\title{
La importancia del razonamiento lógico en la formación del Ingeniero.
}

\author{
Suaza Jimenez, Jorge Hernán \\ Instituto Tecnológico Metropolitano, \\ Colombia \\ jorgesuaza@itm.edu.co
}

\author{
Lora Patiño, Gloria Amparo \\ Politécnico Colombiano Jaime Isaza Cadavid, \\ Colombia \\ glorialora@elpoli.edu.co
}

\section{Resumen}

Los avances científicos y tecnológicos permiten el surgimiento de nuevas formas de ver el mundo y comprender la humanidad; los profesionales enfrentan diversos problemas y situaciones decisivas a las que deben prestar atención, esto exige un pensamiento crítico, desarrollar el autoconocimiento, comprender los problemas y presentar soluciones eficientes y eficaces.

Es indispensable desarrollar habilidades de razonamiento lógico que potencien la capacidad lógico-interpretativa y abstractiva en los estudiantes con el fin de que estos tomen decisiones apropiadas, rápidas y eficientes.

Palabras Clave: Razonamiento lógico; resolución de problemas; lógica; abstracción; pensamiento abstracto;

\section{Abstract}

Scientific and technological advances are allowing the emergence of new ways of seeing the world and understanding the humanity. Professionals are facing different kinds of problems and decisive circumstances to which they must pay attention. That requires a critical thinking, developing of the selfknowledge, problems understanding and be able to present effective and efficient solutions.

It is indispensable to develop logical reasoning skills that improve the logical-interpretative-abstractive capacity in students in order for them to make appropriate, fast and efficient decisions.

Keywords: Logical reasoning; problem solving; logic; abstraction; abstract thinking;

\section{INTRODUCCIÓN}

Uno de los problemas de la educación de hoy es que se continúa dando más importancia a la enseñanza del contenido que al desarrollo de destrezas y habilidades cognitivas que permitan un desarrollo del pensamiento de los estudiantes. La educación contemporánea se enfrenta a nuevos desafíos. Pérez (2010, p. 38) afirma que "La era de la información y de la incertidumbre requiere ciudadanos capaces de entender la 
complejidad de situaciones y el incremento exponencial de la información, así como de adaptarse creativamente a la velocidad del cambio y a la incertidumbre que le acompaña".

Entre las competencias profesionales más relevantes para los ingenieros están el adquirir una disciplina de pensamiento analítico integrando conocimientos de diversa índole, siendo el razonamiento lógico, el modelamiento matemático y el pensamiento abstracto aquellos con mayor nivel de relevancia.

Históricamente, estas competencias han presentado una alta dificultad por su nivel de abstracción, reportando una gran cantidad de repitentes en cada periodo curricular, con altos números de estudiantes que desertan de los programas académicos que se ofrecen. Una forma de lograr este cambio es renunciar al pensamiento lineal con el que se educa desde hace mucho tiempo y comenzar a integrar la práctica y las necesidades e impactos sociales con los conceptos científicos subyacentes. Los retos que presenta la ingeniería actual son multidimensionales por lo que el ingeniero debe tener pleno conocimiento de las dimensiones sociales y políticas para abordarlos; es por ello que se requiere un nuevo tipo pensamiento, que vea la integralidad de los sistemas desde una perspectiva compleja que permita adaptarse y ser parte esencial de un enfoque formal en la comprensión y gestión del conocimiento dinámico y transdisciplinar en la que el razonamiento lógico indique el camino a recorrer para encontrar una solución.

Este artículo es el resultado de una revisión de literatura para determinar la importancia del pensamiento lógico dentro de la formación de los ingenieros en el procedimiento de consulta en diferentes bases de datos y bibliotecas digitales con el objetivo de encontrar trabajos representativos que aportaran a la investigación.

\section{ESTADO DEL ARTE}

EI siglo XXI es una época de grandes cambios, los avances científicos y tecnológicos exigen nuevas habilidades y destrezas en los profesionales que les permita ver el mundo de varias formas. Es por ello 
La importancia del razonamiento lógico en la formación del Ingeniero.

que autores como Futschek (2011), Dagienè (2011) y Ala-Mutka (2004) expresan esfuerzos por dejar atrás las prácticas tradicionales de transmisión de conocimientos de forma magistral, buscando innovar en las formas de transmitir el conocimiento; pero dicha transformación se lleva de forma paulatina.

Bartlett (2001) hace énfasis en que el pensamiento sistémico va a permitir analizar los componentes de una situación determinada de forma global y no aislada, permitiendo retomar el poder de la sencillez y la eficacia del proceso, y a su vez, encontrar el enfoque sistémico en cualquier situación. Al realizarse un adecuado desarrollo, los estudiantes lo pueden utilizar para obtener una perspectiva más profunda acerca de sus habilidades de ingeniería.

Para este autor la gran problemática surge cuando el estudiante no está preparado para superar la discordancia cognitiva de buscar algo antes de saber lo que es. Para ello, es necesario que primero desarrolle el razonamiento lógico, en especial, cuando sus habilidades le deben permitir resolver problemas.
De acuerdo con los autores Felder y Silverman (1988), existen múltiples estilos de aprendizaje y formas de enseñanza en la educación en ingeniería y los profesores, los planes de estudios propios de cada universidad y las didácticas son variantes de acuerdo con las necesidades, las cuales se deben de adaptar a una ciencia cambiante. Estas necesidades tan cambiantes conducen al bajo rendimiento de estudiantes y frustración de los docentes y a una baja capacidad de los profesionales para comprender y solucionar problemas.

Los estilos de aprendizaje son diversos, y la inclusión de un número relativamente pequeño de técnicas didácticas debería ser suficiente para satisfacer las necesidades de gran parte de ellos. Es importante destacar que no hay necesidad de utilizar un sinnúmero de técnicas en cada clase, sino más bien recoger varias que demuestren que son factibles y probarlas en un número considerable de sesiones de clase. No se debe olvidar que todo el proceso y/o técnicas deben estar orientadas a desarrollar habilidades de razonamiento lógico, 
puesto que esta es la base para que el profesional comprenda y abstraiga los problemas que intenta y necesita resolver. Un estilo de enseñanza que sea eficaz para los estudiantes y cómodo para el docente, va a evolucionar de forma natural y relativamente sin contratiempos.

Jonassen, Strobel y Lee (2006) se enfocan en que los estudiantes de ingeniería deberían aprender a solucionar problemas, ya que van a ser contratados para ello. La diferencia radica que la realidad del aula está muy lejos de las necesidades del mundo cambiante. Los autores seleccionados muestran estudios cualitativos de las dificultades propias de la ingeniería en el salón de clases, que son mal elaborados por los educandos debido a que son difíciles. Y los estudiantes presentan conflictos que intentan solucionar con distintos métodos. En general, sus recomendaciones se orientan a trabajar en mayor medida el razonamiento lógico en el aula, tratando de que no esté muy alejados de los problemas que se va a encontrar en su mundo laboral.
Hargadon y Bechky (2006) presentan un modelo de creatividad colectiva, el cual explica cómo en ocasiones las interacciones individuales repercuten al colectivo. EI modelo basado en observaciones, entrevistas, conversaciones y datos de estudio, muestra que a pesar de que algunas soluciones parecieran de una visión individual, en realidad son un proceso de construcción colectivo; es la forma como trabajan la mayoría de los ingenieros hoy en día. Para los investigadores, la creatividad colectiva se refleja en un cambio cualitativo del proceso colectivo como una compensación entre la comprensión del problema y la presentación de soluciones grupales que permitan repetirse en experiencias con anterioridad.

Por otro lado, Scheibler, WiIliams, Mossbrucker, Wrate y Petersen (2006) proponen la estandarización de los productos que evidencian los progresos del aprendizaje de los estudiantes de ingeniería, como por ejemplo, los exámenes, y la reutilización de las lecciones aprendidas de estos, con el fin de guiar el conocimiento adquirido a nuevos métodos y protocolos para lograr una relevancia de 
la tecnología contemporánea en el aula y los laboratorios que se necesitan, es decir, una unión entre la tecnología que el mundo requiere y los laboratorios requeridos para ello.

La educación a nivel de ingeniería ha conservado un equilibrio entre los requisitos técnicos y notécnicos, se retoma la sabiduría popular y la experiencia práctica, lo que permite un mayor énfasis en la capacidad técnica, lo que ha asegurado una profesión operativa. Debido a que los procedimientos cada vez están más alejados de la realidad, es necesario un replanteamiento del desarrollo del razonamiento lógico en las aulas en que se forman los ingenieros. La conclusión de este estudio es que el filtro educativo para la lógica se debe ampliar, para preparar a la siguiente generación de ingenieros para que ingresen al mundo tecnológico con una ventaja competitiva y para que administren adecuadamente los recursos del planeta.

En el trabajo de Pop y Pop (2007) se expone la lógica como uno de los factores más importan- tes en la filosofía, las matemáticas y la informática, y de cómo este puede ser utilizado en la formación de ingenieros para facilitarles el aprendizaje y el desarrollo de habilidades para solucionar problemas y para adaptarse a las nuevas necesidades sociales. Estos autores concluyen en que el desarrollo del razonamiento lógico es un componente necesario en la formación de ingenieros, y que es necesario darle importancia y fortalecer su uso mediante nuevas prácticas, que permitan que los estudiantes se familiaricen con la lógica y puedan aplicarla desde el quehacer diario con el fin mejorar su ejercicio profesional.

Por otra parte, DeWall, Baumeister y Masicampo (2008) enfatizan en que los seres humanos están tiene un potente cerebro que les permite la conciencia y la reflexión, diferenciándonos de otras especies vivientes. Aunque la psicología ha creado una tendencia de cuestionar los beneficios de la conciencia, estos investigadores prueban una hipótesis que estructuran desde cuatro estudios: en el estudio 1 se encuentran una disminución del razonamiento lógico cuando la carga 
cognitiva vuelve a ocupar el razonamiento lógico y obstaculiza el sistema no consciente de pensamientos conscientes; el estudio 2 hace referencia a la activación no consciente de la idea del razonamiento lógico que aumentó la activación de los procesos de la lógica, pero no logra mejorar el rendimiento del razonamiento; en el estudio 3 , a conclusiones lógicas que sean en gran parte intuitivas y no dependan del razonamiento lógico; y en el estudio 4, a la estimulación de una meta consciente da lugar a un mejor rendimiento del razonamiento lógico. Los resultados ofrecen evidencia de que el razonamiento lógico es ayudado por el sistema de procesamiento consciente-reflexivo para desarrollar habilidades en la solución de problemas.

Rodríguez y González (2008) aplican el proceso del razonamiento lógico ( $P R L)$ en la planificación estratégica e interrelacionan cuatro inteligencias: cognitiva, afectiva, operacional y reflexiva, que son innatas del individuo. El programa tuvo una duración de 32 horas y se impartió a 9 estudiantes. El proceso consta de siete etapas: solicitud de audiencia, definición de actividades, diseño del programa, aplicación del programa, instrucción del PRL, aplicación de un nuevo programa y evaluación de los efectos del programa. Los resultados muestran una mejora en la capacidad de análisis y en la capacidad reflexiva de los estudiantes acerca de la resolución de problemas.

Los autores Jeppesen y Lakhani (2009) hacen referencia a formular una solución eficiente, la cual se relaciona directamente con un incremento de la distancia entre el conocimiento y las habilidades de los solucionadores y el campo focal del problema. También, aportan a mejorar, por ejemplo, la teoría del conocimiento empresarial, demostrando la eficacia de un mecanismo de extracción del conocimiento desde diversas fuentes (internas y externas), para resolver los problemas internos. Pero esto solamente será posible si los solucionadores han desarrollado habilidades de razonamiento lógico.

Carmona y Jaramillo (2010) presentan una investigación cuyo propósito es reflexionar sobre el razonamiento lógico y hacen aportes para desarrollarlo en un contexto 
académico en el área de las Ciencias Naturales. Esto lo hacen creando una unidad didáctica enfocada en la resolución de problemas y seleccionan tres estudiantes a los cuales aplican una prueba sicométrica. A partir de allí, desarrollan un plan de observación para aplicarlo en el desarrollo de una unidad respectiva. Al hacer un análisis entre los resultados de inicio y los finales se evidenció un incremento en el nivel de su razonamiento. Si estos investigadores obtuvieron sus resultados en las Ciencias Naturales, es posible adaptar su experimento a procesos en las áreas ingenieriles.

Holvikivi (2010) reporta que los primeros cursos que se imparten en las universidades acerca de la lógica para la programación a menudo no logran motivar a los estudiantes para seguir con los estudios en ingeniería. Esto se debe a que ellos tienen dificultades para desarrollar habilidades lógicas y no están en condiciones de aplicar un razonamiento lógico consistente para seguir instrucciones de manera sistemática. Por otro lado, la transferencia de habilidades de pensamiento lógico desde las matemáticas no logra los resultados esperados. Concluye que existen múltiples dificultades para describir el proceso del desarrollo del razonamiento lógico en la resolución de problemas, por lo que las habilidades son ambiguas. Otro problema es que el conocimiento que se transmite en el aula no es fácil de describir puesto que se basa en el conocimiento experto del profesor y se va acumulando por medio de experiencias y prácticas alejadas de la realidad. Por esto, se requiere una forma de crear patrones mentales apropiados para poder aplicar el razonamiento lógico en la práctica.

Algunos autores como Morin (2010), manifiestan que se debe comenzar a integrar la práctica y las necesidades e impactos sociales con los conceptos científicos subyacentes. Los retos que presenta la ingeniería actual son multidimensionales por lo que el ingeniero debe tener pleno conocimiento de las dimensiones sociales y políticas para abordarlos, es por ello que se requiere un nuevo tipo pensamiento que vea la integralidad de los sistemas desde una perspectiva compleja en la que el razonamiento lógico indique el camino a recorrer para encontrar una solución. 
Para Serna (2011), gran parte de los ingenieros son capaces de producir diseños y soluciones claros y precisos, entre tanto que otros no son capaces. Esto da lugar a la pregunta de si esto será cuestión de inteligencia y si mediante la formación y entrenamiento será posible mejorar en los estudiantes estas habilidades. Este autor explora respuestas a estos interrogantes argumentando que para los profesionales y estudiantes de ciencias computacionales es crucial poseer una buena comprensión de la abstracción, concluyendo que estas áreas del conocimiento deben procurar habilidades de razonamiento lógico en los estudiantes mediante el desarrollo de la capacidad lógico e interpretativa al igual que la abstractiva.

Urbański (2011) presenta en su artículo un análisis de dos conceptos del psicologismo en la lógica: el de Frege y el de Husserl; demostrando, mediante resultados, que ambos están en contra y lucharon contra el nuevo psicologismo, o cognitivismo, porque generaba un gran cambio en la era contemporánea de la lógica. A través del desa- rrollo de temas como la lógica orientada cognitivamente, el investigador indica nuevos campos para el análisis lógico, nuevos métodos y herramientas necesarias para hacerles frente; las bases neutrales del razonamiento lógico y los problemas que existen en la formación de ingenieros. Concluyeron que no hay una forma reglada de enseñar lógica. Las preguntas que giran en torno al psicologismo y a la misma lógica todavía no se responden satisfactoriamente.

Baidowi, Noh y Noh (2013) hablan del nivel de pensamiento de los estudiantes, el cual se puede medir en términos de su capacidad para resolver problemas, lo que necesariamente va a permitir en el estudiante un desarrollo del pensamiento lógico y de las habilidades cognitivas que son necesarias de acuerdo a las necesidades. Esto permite generar hipótesis y abstracciones a nivel de pensamiento, aunque actualmente no se logre el desarrollo suficiente para tener resultados previsibles. Recomiendan que, para desarrollar el nivel de pensamiento y razonamiento lógico, el trabajo en el aula debe ser autónomo y alternativo. Sugieren que el 
estudiante de ingeniería debe desarrollar cierto nivel de habilidades lógicas para comprender y enfrentar los problemas de la vida diaria, y además perfeccionar su razonamiento para hacerles contrapeso a las necesidades que la sociedad sugiere.

Dirksen (2012), básicamente, plantea el funcionamiento del aprendizaje y parte de la hipótesis de que, si algo se repite las veces necesarias, con el tiempo se grabará en la memoria que es a largo plazo; pero advirtiendo que existen excepciones. Hace referencia a que la memoria se basa en la codificación y la recuperación, por lo que los diseñadores de aprendizaje deben pensar en cómo se introduce el material educativo en la memoria a largo plazo, al igual como hacer para que estos recuerden más tarde lo aprendido. Lo que pasa con este estudio es que los estudiantes se encuentran asediados con un flujo constante de información y debido a la falta de dinamismo en el aula se corre el riesgo de que los estudiantes se acostumbren a hábitos poco estimulantes que no permiten el desarrollo de la memoria a largo plazo. Para la mayoría de los do- centes y administrativos, hace falta unir el contexto emocional del aprendizaje con el contexto emocional del estudiante para recordar, la repetición y aprender de memoria información, en la memoria a largo plazo. Lo que no tienen en cuenta es que existen diversos y diferentes tipos de memoria y de estudiantes, por lo que esta es una estrategia limitada.

Daugherty (2012) habla de un modelo específico en el que se identifican varias características para unificar conceptos de ingeniería en los procesos de enseñanza con los estudiantes; entre estos están: comenzar con metas específicas de aprendizaje en la mente e identificar puntos de entrada o "portales" para infundir un verdadero enfoque de ingeniería; modificar o desarrollar el plan de estudios como un desafío ante las necesidades cambiantes del mundo; luego, con la experiencia del aprendizaje de los conceptos infundidos, desarrollarles el razonamiento lógico necesario para comprender el mundo mediante las habilidades necesarias. Otra cuestión, que esta investigación revela, es la importancia de que los docentes tengan la pre- 
paración necesaria para lograrlo: experimentar el plan de estudios. entender los ciclos y los procesos involucrados en el enfoque. aprender el contenido de la ingeniería y experimentar en el aula sin temor al error.

Serna y Serna (2013) son más específicos y hacen un análisis de las necesidades a incluir en lógica en los procesos formativos en a nivel de ingeniería. Los investigadores hacen un recorrido partiendo de la historia de la lógica en estas áreas, luego describen la relación y la necesidad de que esté presente en los procesos de formación relacionados, y al final, hacen un análisis del qué, cuándo y qué tan profundo se deberían de realizar los trabajos en la formación respectiva. Se trata de una revisión al estado del arte y a la importancia de incluirlo en los planes de estudios de los programas ingenieriles. De ello depende que los profesionales estén capacitados para analizar y solucionar los problemas complejos de la sociedad de este siglo.

Otros autores tales como Ghasempour, Bakar y Jahanshahloo (2013) enfatizan en los métodos de enseñanza donde se pueden incorporar actividades metacognitivas y planteamientos para la resolución de problema, implicando que los estudiantes tienen la responsabilidad de encontrar nuevos problemas y reformularlos para encontrar una solución, lo que genera un ambiente de investigación basado en el aprendizaje. También indican que los docentes deberán crear escenarios con situaciones adecuadas, para que los estudiantes los analicen y detecten los posibles problemas. En este trabajo se analizan los resultados de una investigación relacionada con la clasificación de problemas y de las actividades metacognitivas, y en los resultados, se anima a los docentes para que incorporen a los estudiantes en actividades metacognitivas de razonamiento lógico y de resolución de problemas en el aula.

Los autores Serna y Polo (2014) hacen un énfasis entre la relación de la lógica y la abstracción como áreas necesarias en los procesos de formación a nivel de ingeniería destacando la necesidad de la formación en estas áreas del conocimiento y cómo estas son importantes en el ejercicio profesio- 
nal de los ingenieros y en el desarrollo y potencialización de su capacidad lógico-interpretativa $y$ abstractiva para la resolución de cualquier tipo de problema. En esta investigación-revisión-reflexión destaca la importancia de estos, vistos a nivel estructural desde el plan de estudios, concluyendo que solamente con un razonamiento lógico adecuado, los profesionales en ingeniería podrán desarrollar las habilidades necesarias que les demanda el mercado laboral.

Allo (2015) nos habla de la conexión tradicional entre la lógica y el razonamiento lógico que ha estado bajo presión desde que Gilbert Harman propuso que la lógica era una concepción heredada que determina las normas que debemos creer. De esta forma revolucionó todo lo que hasta entonces se entendía de la lógica. El autor desafía a Harman en el contexto más amplio de la dialéctica, con base en revisionistas lógicos como Bob Meyer y algunos escépticos, que han trabajado sobre la importancia de la lógica en el razonamiento. Posteriormente, plantea un modelo formal basado en la epistémica contemporánea y lógica doxástica en el que se pueden cap- turar las relaciones entre la lógica y normas para la creencia. Aunque no trata directamente la relación entre el desarrollo del razonamiento lógico y la formación en ingeniería, las bases conceptuales son válidas para este análisis.

En la investigación de Serna y Serna (2015) se concluye que un asunto importante de la formación de ingenieros es orientar los planes de estudios mediante procesos de detección y resolución de problemas; pero los sistemas de educación ignoran la necesidad de formar en el desarrollo del razonamiento lógico, para que dicha función se desarrolle adecuadamente. En esta investigación se describen los conceptos de la lógica y la resolución de problemas como una necesidad de la formación ingenieril y el desarrollo profesional; además, los autores recomiendan tener en cuenta los requisitos de la información y las necesidades de la sociedad del conocimiento, para ajustar los procesos educativos de los futuros estudiantes formados en ingeniería.

Los estudios anteriores demuestran que la ingeniería es tanto un arte como una ciencia, y para que 
los futuros profesionales de las áreas de ingenierías desplieguen las habilidades, destrezas, y capacidades necesarias para potencializar su ingenio y resolver los problemas de esta sociedad, necesitan desarrollar adecuadamente su razonamiento lógico. Sin embargo, la educación en ingeniería todavía no involucra ampliamente principios como la lógica, la abstracción y la resolución de problemas en los planes de estudios; por otro lado, la fragmentación de los saberes y el aislamiento entre las disciplinas y el uso inadecuado de la tecnología de la información (TIC), genera que los estudiantes no desarrollen estas competencias y por ende no solucionen los problemas de forma adecuada. Gran parte del éxito de los ingenieros es que desarrollen el razonamiento lógico, pero para esto, en las universidades no deben de dejarlo para el final, sino, irlo desarrollando de forma continua, y de ser posible, garantizarlo si se comienza desde las fases primarias de la escuela.

Es evidente que los modelos tradicionales de enseñanza están desapareciendo progresivamente, que las nuevas generaciones de estu- diantes, en calidad de nativos digitales, exigen métodos más adecuados a sus necesidades, que el desarrollo de las clases magistrales no deben ser el común denominador, que el tablero es un componente que debe ajustarse hacia nuevas condiciones y que en el aula ya debe incorporarse otro tipo de componentes como el computador y los dispositivos móviles como una nueva estrategia de instrucción.

\section{AnÁlisis DE RESULTADOS}

De acuerdo con los trabajos analizados en la revisión de la literatura, el estudio del razonamiento lógico se experimenta mediante el descubrimiento de patrones de respuesta difíciles de explicar o que contradicen una teoría ya establecida, luego se crea un modelo que explica el patrón, y, por último, se amplía el modelo para incluir situaciones que engloban fenómenos relacionados. Lo tradicional es que se tiende a investigar un tipo de razonamiento específico, usando una variante de una tarea experimental. Por ejemplo, si se investiga sobre la implicación condicional, se puede aplicar una tarea de selección. Por eso es 
La importancia del razonamiento lógico en la formación del Ingeniero.

que los experimentos tienden a descubrir un patrón de razonamiento, como por qué los estudiantes tienden a seleccionar opciones ya establecidas en lugar de generar sus propias respuestas. Esto se refleja cuándo quedan expuestos a una situación en la que deben resolver cierto tipo de problema. En la mayoría de casos, optan por resolverlo abordándolo de diferentes maneras, o planteando diferentes estrategias que puedan darle solución y es poco común que desarroIlen un estándar de solución que puedan reutilizar cuando sea necesario.

Los resultados en los estudios han encontrado una variación individual en el rendimiento del desarrollo de la lógica el cual se relaciona con la capacidad de llevar a ejecución ciertas tareas mas no otras. Para Carmona y Jaramillo (2010), al aplicar el modelo que proponen, se podría predecir cómo la elección de una estrategia de solución podría variar con el desarrollo del razonamiento lógico, y con el tiempo, esa estrategia se podría reutilizar para comprender los resultados de una clase de fenómenos de razonamiento tales como la habilidad de dedu- cir de forma independiente una tarea ya sea por la experiencia o por la edad; a partir de estos se hace posible unificar una teoría. Por otro lado, en la medida que las estrategias para la solución de problemas, y los problemas mismos, se vuelven más complejos, se necesita desarrollar capacidades lógicas avanzadas que, de acuerdo con el tipo de problema, permitan seleccionar la mejor estrategia de solución, es decir, una estrategia lógica.

Debido a que el razonamiento lógico es no-cuantitativo, no se puede medir fácilmente; no existe una medida estándar que pueda indicar con precisión en qué grado se encuentra su desarrollo en una persona, pero existen varios factores que podrían indicar el grado del desarrollo del razonamiento lógico que una persona tiene en determinado momento. Algunos autores consideran que la argumentación es uno de ellos debido a que el diálogo argumentativo exterioriza el razonamiento argumentativo, es decir, no hay forma de conocer exactamente lo que ocurre en el interior de la mente, pero una de las formas en que podemos aproximarnos es 
prestando atención a las discusiones, por ejemplo, entre estudiantes, acerca de cuestiones científicas.

En este caso se centraron en la forma cómo deberían resolver los problemas propuestos, convirtiéndose en el conjunto de enunciados que ellos formulan, y que, aunque estas no sean totalmente ciertas, van a permitir construir el conocimiento y las expresiones que son relacionadas con el razonamiento lógico. En este mismo sentido, se puede recomendar que, al realizar un análisis de un discurso argumentativo sobre cuestiones polémicas en lenguaje natural, se requiere, entre otras cosas, prestar atención al lenguaje mismo y ser capaz de analizar proposiciones relativamente ambiguas o vagas. Además, los investigadores en este tema deben estar preparados para desenredar la línea fundamental de argumentación en medio de extensos intercambios entre dos o varias personas, porque en una conversación de quienes realmente conocen y saben desenvolverse en un campo específico de la ciencia, sus argumentaciones y expresiones detonan su grado de conocimiento $y$ cuando ese conocimiento es muy arraigado, lo pueden expresar cotidianamente y hacerlo entendible para quienes los escuchan y que pueden no estar al tanto de ello. Esta forma de expresión es el reflejo de un razonamiento lógico en alto desarrollo, y puede servir como modelo para medir su grado.

Para otros, el valor de razonamiento lógico depende en parte del hecho de que los estudiantes pueden compartir lo que entiende con base a ciertas reglas $y$, por tanto, corregirse unos a otros argumentando los errores que consideran en los demás. La cuestión es que la mente humana puede llevar de mejor manera los procesos sistémicos o conscientes que aquellos que son automáticos 0 inconscientes. Otra forma de poder interpretar esto es cuando se concibe la conciencia humana como la propiedad de un segmento de la mente individual, pero a largo plazo, puede ser necesaria una dimensión interpersonal para alcanzar una comprensión completa de las situaciones que nos rodean. Mientras las personas sean más conscientes de su razonamiento, pueden interiorizar mejor el conocimiento, y así, transmitirlo de adecuadamente a otras o em- 
plearlo para solución o resolución problemas. A esto se debe que muchos de los autores analizados concluyan que, antes de desarrollar el razonamiento lógico, los estudiantes deben ser lógicos, utilizar la lógica en la vida cotidiana y usarla como una herramienta en cualquier ámbito que les toque vivir.

Cuando los estudiantes desarroIlan su capacidad lógico-interpretativa y abstractiva, se puede afirmar que llegaron a la escala del dominio de la lógica. Esto se puede considerar como el primer peldaño en la escalera que los conduce hacia el razonamiento lógico. Los estudiantes de ingeniería son un caso particular de este determinismo porque necesariamente están sujetos e inmersos a aplicar su ingenio para comprender y solucionar problemas. No se trata solamente de memoricen 0 interioricen conceptos y teorías sino de que desarrollen un razonamiento lógico que les permita ubicarse en el mundo ingenieril que eligieron. Esto quiere decir que serán profesionales con habilidades especiales para abstraer y modelar soluciones eficientes y efectivas.

\section{CONCLUSIONES}

Una definición ampliamente aceptada para razonamiento lógico es la que se asocia a las operaciones lógicas del pensamiento, como proceso racional del cerebro que permite llegar a las conclusiones correctas. Para esto se han llevado a cabo experimentos en los cuales se evalúa la capacidad de las personas para resolver problemas (Carmona y Jaramillo, 2010; Serna y Serna, 2015). Otros autores divulgan acerca de cómo reaccionan los estudiantes ante diferentes situaciones y problemas, que han llevado a que las instituciones modifiquen los programas educativos para que estos actores tengan una mejor visión de lo que representa resolver problemas, y a la vez para que comprendan el mundo de otra manera y puedan aplicar ese conocimiento en su mejoramiento (Felder y Silverman, 1988; Allo, 2015).

La mayoría de estas investigaciones giran en torno a áreas del conocimiento en las que predomina la lógica, tales como las Ciencias Computacionales, sicología, neurociencias y neurocomputación; y su 
objetivo es que los participantes aprendan a desarrollar o potencializar un pensamiento crítico que les ayude a mejorar sus destrezas, habilidades y capacidades para solucionar problemas (Pop y Pop, 2007; Urbański, 2011). Entre los estudios revisados se puede citar al de Baidowi, Noh y Noh (2013) quienes establecieron dos programas en Ingeniería y Ciencias, a los cuales solamente podían ingresar los estudiantes que alcanzaran el puntaje más alto en ciertas asignaturas. De 700 aspirantes, 145 completaron correctamente las respuestas del examen. Al evaluar los resultados, los autores observaron que, aunque los estudiantes demostraban un alto conocimiento de las materias y entendían las temáticas relacionadas, no mostraban un razonamiento lógico adecuado que les permitiera solucionar los problemas planteados.

Por su parte, Holvikivi (2010) reporta el caso de un grupo de estudiantes de técnicas de programación, a los que se les enseñaron múltiples estrategias sobre Ciencias Computacionales, incluyendo herramientas de aprendizaje, didácticas y métodos pedagógicos.
Este investigador observó que el nivel de aprendizaje de los participantes en cada uno de los cursos era bajo, en especial en lo relacionado con el desarrollo de habilidades matemáticas. Su conclusión fue que el desarrollo del razonamiento lógico necesario era afectado por factores tales como la cultura, la región de donde provenían y los conocimientos adquiridos en sus estudios previos.

Estos estudios revelaron que el desarrollo del pensamiento lógico solamente es posible cuando se comienza a trabajar desde las etapas iniciales del proceso formativo. Además, el resultado es que, cuando este razonamiento no se desarrolla adecuadamente, es complejo aprender a resolver problemas (Holvikivi, 2010).

Entonces, ¿cuál es la relación entre el razonamiento lógico y el desarrollo de destrezas, habilidades y capacidades ingenieriles? Al desarrollo de la lógica también se unen habilidades más específicas propias de toda labor del ingeniero tales como realizar operaciones lógicas entre ellas las inferencias, la 
La importancia del razonamiento lógico en la formación del Ingeniero.

deducción, abstracción, refutación, la argumentación y la demostración directa e indirecta (Carmona y Jaramillo, 2010). Esto se debe a que, en esta profesión, la lógica es un tema fundamental que se utiliza en la vida diaria. Al aprender, estudiar y razonar acerca de los conocimientos, hechos, hipótesis y reglas de deducción, en realidad se utiliza la lógica, e incluso la falta de lógica indica algún tipo de lógica (Pop y Pop, 2007).

El trabajo que realizan los ingenieros consiste en detectar, comprender y solucionar problemas, por ello deben cultivar el razonamiento lógico y desarrollar una capacidad lógico-interpretativa y abstractiva que les ayude a tomar decisiones apropiadas, de manera rápida y eficiente (Serna y Serna, 2015; Jonassen, Strobel y Lee, 2006). De ahí la importancia de la lógica y del razonamiento lógico en la formación y el desarrollo profesional de los ingenieros, porque de esta manera, son capaces de ampliar su conocimiento y comprensión y desarrollan el auto-conocimiento, comprenden los problemas y presentan soluciones eficientes y eficaces. En este sentido, es necesario que desarrollen capacidades para reflexionar acerca de las funciones cognitivas y de las habilidades meta-cognitivas relacionadas con la lógica (Serna y Flórez, 2013).

\section{BIBLIOGRAFÍA}

Ala-Mutka, K. (2004). Problems in Learning and Teaching Programming. Finlandia, Tampere University of Technology. Institute of Software Systems: Codewitz Needs Analysis, 1-13.

Allo, P. (2015). Logic, Reasoning and Revision. Theoria, 82(1), 3-31. doi: $10.1111 /$ theo. 12071

Baidowi, Z., Noh, N., \& Noh, N. (2013). A Study on the Significance of Students' Thinking Level to Students' Performance. Procedia - Social and Behavioral Sciences, 90, 914-922. doi: 10.1016/j.sbspro.2013.07.168

Bartlett, G. (2001). Systemic thinking: A simple thinking technique for gaining systemic focus. En The International Conference On Thinking "BreakThroughs 2001" (pp. 1-14). Los Angeles, USA.

Carmona, N., \& Jaramillo, D. (2010). El razonamiento en el desarrollo del pensamiento lógico a través de una unidad didáctica basada en el enfoque de resolución de problemas (Tesis de Maestría). Universidad Tecnológica de Pereira. Facultad de Ciencias de la Educación. Maestría en Educación. Recuperado de WorldCat Discovery, Catálogo Bibliográfico en Línea de la Universidad Tecnológica de Pereira https://utp.on.worldcat.org/oclc/991684364 
Dagienè, V. (2011). Informatics Education for New Millennium Learners. En: Kalas, I., \& Mittermeir, R. (eds). Informatics in Schools. Contributing to 21st Century Education. Berlin, Heidelberg:

Springer Berlin Heidelberg. Lecture Notes in Computer Science, 7013, 9-20. doi: 10.1007/978-3-642-24722-4_2

Daugherty, J. (2012). Infusing engineering concepts: Teaching engineering design. (Reporte de Investigación). Utah State University. DigitalCommons@USU: Publications. 170, 1-12. Recuperado de https://digitalcommons.usu.edu/ncete_publications/170/

DeWall, C., Baumeister, R., \& Masicampo, E. (2008). Evidence that logical reasoning depends on conscious processing. Consciousness and Cognition, 17(3), 628-645. doi: 10.1016/j.concog.2007.12.004

Dirksen, J. (2012). Design for how people learn. Berkeley, California: New Riders.

Felder, R., \& Silverman, L. (1988). Learning and teaching styles in engineering education. Engineering education, 78(7), 674-681.

Futschek G., \& Moschitz J. (2011). Learning Algorithmic Thinking with Tangible Objects Eases Transition to Computer Programming. En: Kalas, I., \& Mittermeir, R. (eds). Informatics in Schools. Contributing to 21st Century Education. Berlin, Heidelberg: Springer Berlin Heidelberg. Lecture Notes in Computer Science, 7013, 155-164. doi: 10.1007/978-3-64224722-4_14

Ghasempour, Z., Bakar, M., \& Jahanshahloo, G. (2013). Innovation in Teaching and Learning through Problem Posing Tasks and Metacognitive Strategies. International Journal of Pedagogical Innovations, 1(1), 53-62. doi:

10.12785/ijpi/010108
Hargadon, A., \& Bechky, B. (2006). When Collections of Creatives Become Creative Collectives: A Field Study of Problem Solving at Work. Organization Science, 17(4), 484-500. doi: 10.1287/orsc. 1060.0200

Holvikivi, J. (2010). Conditions for Successful Learning of Programming Skills. In Reynolds, N., \& Turcsányi-Szabó, M. (eds). Key Competencies in The Knowledge Society. Berlin, Heidelberg: Springer Berlin Heidelberg. IFIP Advances in Information and Communication Technology, 324, 155-164. doi: 10.1007/978-3642-15378-5_15

Jeppesen, L., \& Lakhani, K. (2009). Marginality and problem-solving effectiveness in broadcast search. Organization Science, 20, 1-44. Recuperado de https://dash.harvard.edu/handle/1/3351241

Jonassen, D., Strobel, J., \& Lee, C. (2006). Everyday Problem Solving in Engineering: Lessons for Engineering Educators. Journal of Engineering Education, 95(2), 139-151. doi: 10.1002/j.2168-9830.2006.tb00885.x

Morin, E. (2010). Sobre la Interdisciplinariedad. Centre International de Recherches et Etudes Transdisciplinaires (CIRET), 2.

Pérez, Á. (2010). Aprender a educar. Nuevos desafíos para la formación de docentes. Revista Interuniversitaria de Formación del Profesorado, $68(24,2)$, 37-60

Pop, H., \& Pop, L. (2007). Education for engineering students - The case of logic. En Proceedings 6th International Conference on Electromechanical and Power Systems (pp. 259-264). Chisinau, Moldavia. 
Rodríguez, C., \& González, S. (2008). El proceso de razonamiento lógico y la enseñanza de la planificación estratégica. Actualidad Contable FACES, 11(17), 111-121. Recuperado de https://biblat.unam.mx/es/revista/actualidad-contable-faces/articulo/el-proceso-de-razon amiento-logico-y-la-ensenanza-de-la-planificacion-estrategica

Scheibler, S., Williams, S., Mossbrucker, J., Wrate, G., \& Petersen, O. (2006).

Creating A "Global Algorithm" For Engineering Education. En 2006 Annual Conference \& Exposition (pp. 1-11). Chicago, Illinois. Retrieved from https://peer.asee.org/1011

Serna, E. (2011). Abstraction as a critical component in Computer Science training. Avances en Sistemas e informática, 8(3), 79-83.

Serna, E., \& Flórez, G. (2013). El razonamiento lógico como requisito funcional en ingeniería. En Eleventh LACCEI Latin American and Caribbean Conference for Engineering and Technology (LACCEl'2013) (pp. 1-10). Cancún, México. Recuperado de http://www.laccei.org/LACCEI2013-Cancun/RefereedPapers/RP221.pdf

Serna, E., \& Serna, A. (2013). Logic in Computer Science. Revista Educación en Ingeniería, 8(15), 62-68.

Serna, E., \& Polo, J. (2014). Logic and abstraction in engineering education: $A$ necessary relationship. Revista Ingeniería Investigación y Tecnología XV (2), 299-310. Recuperado de http://www.scielo.org.mx/scielo.php?script=sci_arttext\&pid=\$1405$77432014000200012 \&$ Ing=es\&tlng=es.
Serna, E., \& Serna, A. (2015). Knowledge in Engineering: A View from the Logical Reasoning. International Journal of Computer Theory and Engineering, 7(4), 325-331. doi: 10.7763/ijcte.2015.v7.980

Urbański, M. (2011). Logic and cognition. Two faces of psychologism. Logic and Logical Philosophy, 20(1-2), 175-185. doi:10.12775/LLP.2011.009 